\title{
Research on Marketing Prediction Model Based on Markov Prediction
}

\author{
Haiying Chen, ${ }^{1}$ Haiyan Chen, ${ }^{2}$ Wei Zhang, ${ }^{1}$ Chaodan Yang $\mathbb{D},{ }^{3}$ and Hongxiu Cui $\mathbb{D}^{1}$ \\ ${ }^{1}$ Business School of Changchun Sci-Tech University, Changchun, Jilin 130600, China \\ ${ }^{2}$ Bone Injury Hospital of Siping City, Siping, Jilin 136000, China \\ ${ }^{3}$ Economic Management Teaching and Research Department of Jilin Provincial Party School, Changchun, Jilin 130012, China
}

Correspondence should be addressed to Hongxiu Cui; 100231@cstu.edu.cn

Received 26 October 2021; Revised 16 November 2021; Accepted 17 November 2021; Published 15 December 2021

Academic Editor: Xin Ning

Copyright (c) 2021 Haiying Chen et al. This is an open access article distributed under the Creative Commons Attribution License, which permits unrestricted use, distribution, and reproduction in any medium, provided the original work is properly cited.

\begin{abstract}
Many activities in modern business marketing management are random and repetitive. The marketing effect is constantly influenced by a variety of factors such as changing market supply and demand, customers' purchase intentions, and national financial policy. As a result, Markov analysis can be used to analyze the status and trend of some variables, that is, to predict the future status and trend of a variable based on its current status and trend, in order to forecast possible changes in the future and take appropriate countermeasures. The mathematical model of product marketing prediction is presented in this paper by establishing the probability matrix of product state transition and analyzing and calculating with the Markov chain, resulting in a practical and reliable theoretical basis for economic prediction. After using the Markov analysis method, a suitable mathematical model can be created based on market investigation and statistics, which is extremely useful for making reasonable predictions about the market's future development trend and improving marketing effectiveness.
\end{abstract}

\section{Introduction}

The changing relationship between market supply and demand, customers' purchase intention, national financial policy, and other factors is constantly affecting the effect of marketing. The market situation will continue to be affected by various aspects of the market environment and other factors, including many complex factors and the comprehensive impact of complex subsystems [1]. The impact of market situation transformation on marketing effect seems to be a random phenomenon that cannot be mastered, but the constantly changing information of market information can be collected and its reasonable change law can be obtained through mathematical analysis [2]. Marketing prediction is an important part of marketing planning. Marketing prediction is a series of plans for the development of future customer groups, including the composition of customer groups, the development prediction of future customer groups, and the optimization strategy and value promotion of customer groups in order to maximize profits [3]. There are always these changes in customer value, some increase, some decrease, and even loss. How to grasp the law of customer change and predict the development direction of customer base according to these changes is not only the content that enterprises need to understand in marketing prediction but also the guarantee for the long-term development of enterprises [4]. The market situation will change with the change of the market environment. Although this change is unpredictable, we can get its reasonable change law according to the collected information leading to the market change [5].

Prediction is people's prior inference or judgment of the future or unknown situation of things based on historical data and reality and using the mastered knowledge and means. Its purpose is to provide basis for planning or decision-making [6]. As a general methodology, prediction theory can be applied to the study of both natural and social phenomena. The Markov process is a branch of modern 
probability theory that deals with stochastic processes. It has been widely used and played an important role in many fields, including communication, control, biology, social science, and other scientific and technological fields [7]. In practice, people frequently come across a random process that has the following characteristics: its future evolution is independent of its previous evolution when its current state is known. The independent characteristic of "future" and "past" under the condition of known "present" is called Markov process, and the random process with this property is called Markov process [8]. Markov analysis is a method for predicting the future state of things by analyzing the evolution trend and state of a Markov chain [9]. We do not need a lot of statistical data to predict the future with this method; instead, we only need recent data. The creation of a Markov prediction model can be used to analyze the main factors influencing marketing effect and their mathematical implications [10]. So that the marketing campaign can be scientific, systematic, and modelled.

Customers are the source of enterprise income, a stable customer base is the guarantee for enterprise survival and development, and enterprise marketing prediction is one of the important work of enterprise marketing [11]. The change of the market can be regarded as a random phenomenon. For different market environments, it is said that the system is in different states. Therefore, using Markov chain model to predict market share is mathematically reasonable and feasible [12]. The customer factor has become an important factor in the economic and social environment where the enterprise is located. The enterprise's understanding of the customer situation determines whether the enterprise can survive to some extent [13]. These companies are also studying and understanding the value of each customer, so as to know what is worth investing money and energy to maintain the relationship, improve the enterprise income and profit, and who should give up, so as to reduce the sales cost and avoid the unnecessary loss of profits [14]. In this paper, the Markov chain model is used to predict the change trend of customers and formulate the marketing prediction strategy suitable for the actual situation of enterprises. After using the widely used Markov analysis method in engineering, we can create an appropriate mathematical model based on market research and statistics and make a reasonable prediction of the market's future development trend, which is extremely useful for improving marketing effectiveness.

This paper begins by discussing the importance of marketing prediction and presenting the feasibility of using a Markov model. It then presents the basic model of Markov model prediction and analyzes its application mode. Finally, when combined with the simulation results, it highlights the algorithm's benefits.

\section{Related Work}

The theory of stochastic processes plays a significant role in modern statistics, and the Markov chain is one of the most comprehensive stochastic process theories, with applications in geology, biology, economics, water resources science, atmospheric science, and other fields. Markov has become increasingly important in a variety of random variables, including speech signals, digital image signals, and market trends. The Markov chain is used in commodity sales forecasting, business profit forecasting, and market share forecasting.

In economic forecasting methods, Markov forecasting does not need a large amount of historical data, but only needs recent data to predict the future. Literature [15], according to the characteristics of commodity sales, establishes a Markov forecasting model which can predict commodity sales, thus, providing theoretical basis for enterprises to formulate commodity sales strategies. Literature [16] studies Markov chain and its application in economic forecasting and discusses how to minimize investment and maximize profits in the process of product sales. Nonperforming loans are the focus and core of credit risk management in commercial banks. Literature [17] makes an empirical analysis of loan risk by using Markov model and points out that Markov chain is operable and effective in loan risk analysis, which can be used for forecasting and risk management of bank credit assets. The annual loan migration matrix can effectively analyze the changing characteristics of loan risk and the inherent laws of loan loss and recovery and can measure the multifaceted effect of nonperforming loan divestiture on loan risk transformation, which can provide a basis for banks to take risk prevention measures in advance and improve loan quality [18]. Literature [19] uses Markov chain state transition matrix to analyze and predict bank overdue loans and uses Markov chain method to dynamically monitor bank overdue loans, which has certain reference value for banks to effectively manage overdue loans. Regarding the market price of the securities market and the time series of changes in various returns as a Markov chain, the transition probability can be used to predict the future state according to the current state, and corresponding strategies can be adopted [20]. Literature [21] applies the Markov process to fund investment and obtains a method of inferring the market price trend of the fund from the deviation degree of the discount rate from the value center to determine the timing of buying and selling, which provides a scientific reference basis for investment decision-makers. Literature [22] tried to use the Markov chain model to conduct an empirical analysis of the future trend of the RMB exchange rate, established a Markov chain model, and concluded that the current RMB exchange rate depreciation is very unlikely; on the contrary, there will be a relatively large appreciation in the future. Literature [23] regards the number of employees as a set of random variables that changes over time and uses Markov models to analyze and predict them.

The Markov model can predict internal human resource supply by comprehensively predicting internal personnel transfer, which is advantageous for managers to consider various influencing factors and systematically consider internal personnel promotion, demotion, and turnover. This paper uses this information to create a state transition probability matrix for products, analyzes and calculates it using a 
Markov chain, and presents a mathematical model for product marketing prediction that can be used for enterprise marketing management and market prediction to forecast market share and customer purchasing power trends.

\section{Significance of Marketing Forecast}

In 1985, American Marketing Association defined marketing management as marketing practice is a process of planning and implementing ideas, designing, pricing, promoting, and distributing goods and services, and creating exchange opportunities to meet customer needs and organizational goals. It can be seen that marketing management is a process including analysis, planning, execution, and control. On the basis of exchange, the goal is to meet the needs of all relevant subjects. Because the prediction can estimate the external events that cause fluctuations in a certain phenomenon, we have a certain understanding of the future development of this phenomenon, so that when formulating marketing strategies, we can achieve clear objectives, specific tasks, and practical feasibility. Marketing should affect demand level, demand time, and demand composition, and finally, realize demand management. Forecasting [24] plays a particularly important role in marketing management. In modern management, scientific decision-making occupies a key position, and whether the decision-making is scientific or correct depends not only on the understanding of the present situation but also on the prospect and prediction of the future.

The globalization of the market makes the market competition more intense, and the customer's needs are more diversified and personalized. These requirements require the use of appropriate mathematical models and methods to make a more scientific prediction of various economic indicators related to the market and to conduct quantitative research on the economic activities of enterprises, so as to make correct decision-making plans for marketing managers. With the continuous improvement of people's living standards and economic strength, the correct results of marketing prediction will help families and individuals to grasp the investment direction, manage their finances rationally, and invest correctly. Markov chain model can solve complicated and changeable marketing problems by applying the relevant theories of Markov chain, and through the construction of transition probability matrix and matrix operation, and is widely used in the practice of marketing management.

\section{Markov Analysis Prediction}

4.1. Markov Chain. Markov analysis method takes the system state transition diagram as the analysis object and analyzes the discrete stable state or continuous time changing state of the system subject to a given state transition rate. Markov prediction technology is a technology that applies the basic principles and methods of Markov chain to study and analyze the changing rules of time series and predict its future changing trends. In this model, the continuous time variation of the system is divided into several states to represent the working modes at different times. If the things studied are collectively referred to as systems, Markov analysis method is a dynamic stochastic mathematical model based on the concepts of system "state" and "state transition" [25]. The so-called state is the smallest set of variables representing the system. When a set of variable values is determined, the system is said to be in a state. The socalled state transition means that when a system variable changes from one specific value to another, it means that the system is transferred from one state to another, thus, realizing the system state transition.

Assuming that there are states of $N$ variables, then, one step transition from state $i$ to $j$ is possible, and $P_{i j}$ is called the one-step transition probability. Arrange these probabilities in turn to form a matrix called the state transition probability matrix, denoted as $P$ :

$$
P=\left(\begin{array}{cccc}
p_{11} & p_{12} & \cdots & p_{1 n} \\
p_{21} & p_{22} & \cdots & p_{2 n} \\
& \cdots \cdots & \\
p_{n 1} & p_{n 2} & \cdots & p_{n n}
\end{array}\right) .
$$

The state transition probability matrix $P$ has the following properties: each element of the matrix is nonnegative, and the sum of the elements of each row of the matrix is equal to 1 .

The corresponding element in $P$ is the one-step transition probability, and similarly, multiple transition probabilities can be derived. If the system is in state $i$ at time, after $n$-step transition, it is in state $j$ at time. The quantitative index of the possibility of this transition is called the $n$-step transition probability, which is recorded as

$$
P\left(X_{n}=j \mid X_{0}=i\right)=P_{i j}(n) .
$$

The $N$-step transition probability matrix is denoted as

$$
P=\left(\begin{array}{cccc}
p_{11}(n) & p_{12}(n) & \cdots & p_{1 n}(n) \\
p_{21}(n) & p_{22}(n) & \cdots & p_{2 n}(n) \\
& \cdots \cdots & & \\
p_{n 1}(n) & p_{n 2}(n) & \cdots & p_{n n}(n)
\end{array}\right) .
$$

After calculation, a useful conclusion can be obtained: $P(n)=N$ factorial of $p$. In addition, the $n$-step transition probability has the same properties as the one-step transition probability: each element of the matrix is nonnegative, and the sum of the elements of each row of the matrix is equal to 1 .

\subsection{State Space Classification Method}

4.2.1. Sample Mean Square Deviation Classification Method. The traditional method of index value classification is to use the sample mean and the sample mean square error to describe the change interval of the index value. Suppose 
the index value sequence is $x_{1}, x_{2}, \cdots, x_{n}$, the sample mean is $\bar{x}$, and the sample mean square error is

$$
s=\sqrt{\frac{1}{n-1} \sum_{i=1}^{n}\left(x_{i}-\bar{x}\right)^{2}} .
$$

According to the specific situation, the sample mean is centered and the sample mean square error is used as the standard to group. In application, the index value is often divided into five groups, and the change interval of the index value can be expressed as

$$
\begin{gathered}
\left(-\infty, \bar{x}-\alpha_{1} s\right),\left(\bar{x}-\alpha_{1} s, \bar{x}-\alpha_{2} s\right) \\
\left(\bar{x}-\alpha_{2} s, \bar{x}+\alpha_{2} s\right),\left(\bar{x}+\alpha_{2} s, \bar{x}+\alpha_{1} s\right),\left(\bar{x}+\alpha_{4} s,+\infty\right) .
\end{gathered}
$$

$\alpha_{1}$ can take a value in $[1.0,1.5]$, and $\alpha_{2}$ can take a value in $[0.3,0.6]$.

Using this method to classify index values does not consider the influence of physical causes on index values, but simply takes the sample mean as the center of index values from a statistical point of view, which is somewhat farfetched in terms of scientificity and rationality, but it is more convenient to operate.

4.2.2. Ordered Clustering Method. Ordered clustering is a classification method for ordered samples. When using ordered clustering to divide the change interval of index values, the data structure of the index value series is taken into account fully, making the divided interval more reasonable. In fact, the statistical index values are arranged in a specific order in many practical problems, and this order cannot be disturbed when classifying [26]. Due to the addition of the order constraint, the classification method for this type of ordered statistical index values has its own distinct style.

The classic algorithm for ordered clustering is Fisher's algorithm, which is the optimal segmentation method. Assume that the index value is $x_{1}, x_{2}, \cdots, x_{n}$ in turn, where each $x_{1}$ is a $p$-dimensional vector. Suppose a certain class of $G_{i j}$ is $\left\{x_{i}, x_{i+1}, \cdots, x_{j}\right\}$, and $j \geq i$ defines its mean vector as

$$
\bar{x}_{i j}=\frac{1}{j-i+1} \sum_{l=i}^{j} x_{l} .
$$

The total difference between the index values within the class is defined as the diameter of the class. Since the most commonly used quantitative indicator to express the total difference between index values is the sum of squared deviations, the commonly used diameter is the sum of squared deviations within the class. Using $D(i, j)$ to represent the diameter of $G_{i j}$, then the definition:

$$
D(i, j)=\sum_{l=i}^{j}\left(x_{l}-\bar{x}_{i j}\right)^{T}\left(x_{l}-\bar{x}_{i j}\right) .
$$

Divide $n$ ordered index values into $k$ categories, and set a certain classification method as

$$
p(n, k):\left\{x_{i_{1}}, \cdots, x_{i_{2}-1}\right\},\left\{x_{i_{2}}, \cdots, x_{i_{3}-1}\right\}, \cdots,\left\{x_{i_{k}}, \cdots, x_{n}\right\} .
$$

Among them, the subpoint $1=i_{1}<i_{2}<\cdots<i_{k}<i_{k+1}=n$ defines the error function of this classification, that is, the objective function is the sum of squares of the total deviation within the class:

$$
e[p(n, k)]=\sum_{j=1}^{k} D\left(i_{j}, i_{j+1}-1\right) .
$$

Theoretically, it can be proved that the so-called most significant division is a division method $e[p(n, k)]$ when the minimum value is reached. As for the determination of the classification number $k$, you can use $e[p(n, k)]$ to make a graph of the relationship with $k$, and the $k$ value at the curve corner is the optimal classification number.

4.3. Application of Markov Model in Marketing Forecast. Markov analysis has an important application in the study of random events and corresponding random variables. In the process of marketing, the change of the supply of market commodities is random due to the influence of various uncertain factors. And the marketing personnel should be able to predict the future development trend of the market according to the current market situation, so as to adopt the correct marketing plan [27]. When choosing between marketing effect and marketing cost, Markov analysis method can be used for optimization analysis. Influenced by various factors in the external competitive environment, the value of customers to the enterprise is constantly changing, and thus the enterprise customers are composed of customers with different values. The theoretical model of customer behavior intention is shown in Figure 1.

Enterprises can subdivide customers according to different customer values. Many factors that affect customer values include customer stability, customer satisfaction, and contribution to enterprises, including contribution to enterprise profits, share, and contribution to goodwill. Through analysis, when the market situation is in a stable state, it is generally unnecessary to spend more competitive costs. Compared with the established Markov analysis model, we should not only pay attention to the analysis of the market stability but also constantly pay attention to the analysis of marketing effects.

Facing the market environment of diversified customer sources and diversified customer value preferences, we must capture this market signal as timely as possible and catalog it according to the customer's consumption characteristics and preferences. The data mining process in customer analysis is shown in Figure 2.

According to the transaction data and the user's evaluation information, the user's preference for each car product 


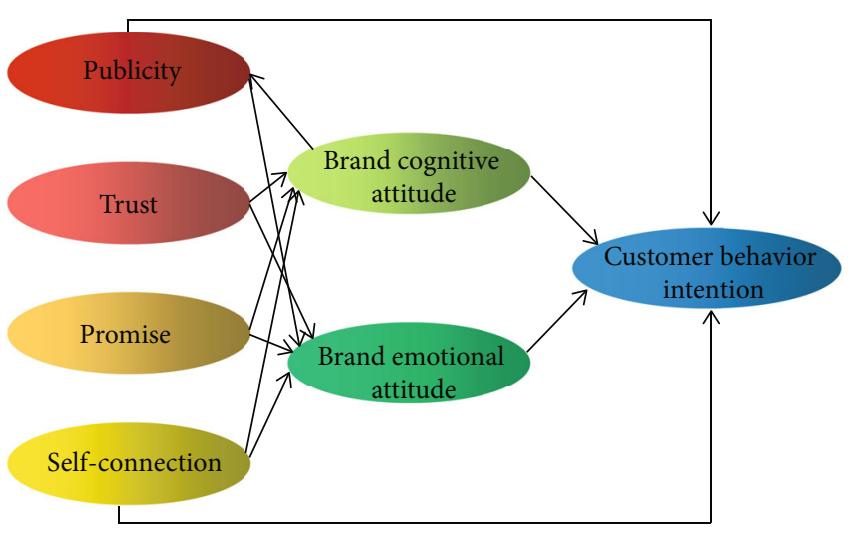

Figure 1: Theoretical model of customer behavior intention.

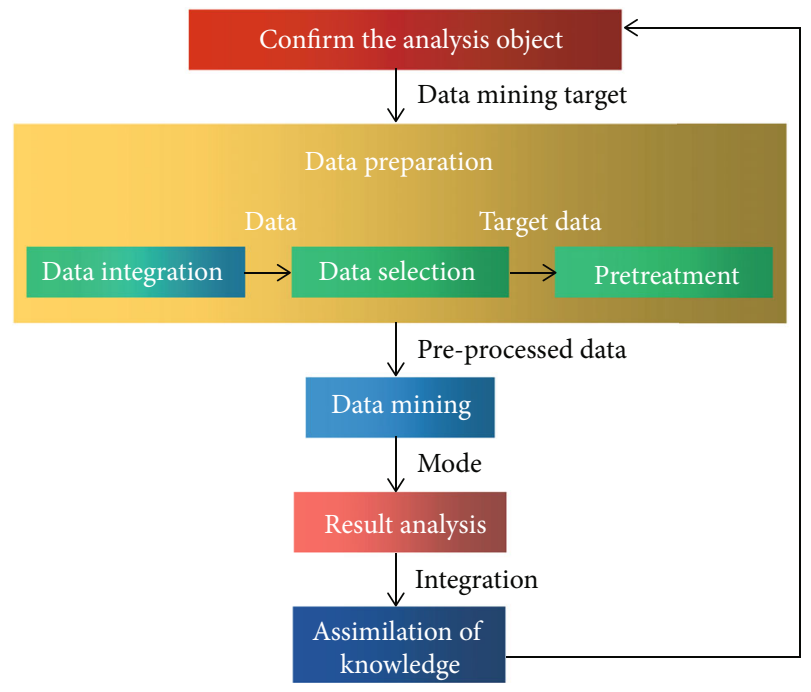

FIGURE 2: Data mining process.

category can be calculated:

$$
P C_{u, j}=\frac{\sum_{i \in I_{u}} P I_{u, i} \times \mu_{j}\left(x_{i}\right)}{\sum_{i \in I_{u}} \mu_{j}\left(x_{i}\right)} j=1,2,3 \cdots .
$$

In the formula, $P C_{u, j}$ represents user $u$ 's preference value for category, $P I_{u, i}$ represents user $u$ 's rating value for product $i, I_{u}$ represents the set of products that user $u$ has evaluated, and $\mu_{j}\left(x_{i}\right)$ represents the degree of membership of product $i$ to category $j$.

The recent customer satisfaction with the company is linked to customer change. Customer satisfaction is, of course, a result of a variety of factors, but it has nothing to do with the previous status of enterprise satisfaction. As a result of the Markov chain, we can predict the change of enterprise customers, providing a reference for enterprise marketing prediction. We strive to increase the types and specifications of products in the appearance packaging of products in order to facilitate customers' portability and choice. In the marketing process, we should constantly stimulate customers' interest in purchasing products. It is necessary to constantly adjust the marketing strategy according to the changes of the market and to adjust the sales strategy according to the changes of the market and the current situation in order to continuously improve the marketing effect.

\section{Result Analysis and Discussion}

Sales forecast refers to estimating the sales quantity and sales amount of the whole product or a specific product in a specific time span in the future. On the basis of fully considering all kinds of influencing factors in the future, combining with the sales performance of the enterprise, feasible sales targets are put forward through certain analysis methods. In the process of marketing, the market conditions that affect the marketing effect are complex and changeable random phenomena, that is to say, various factors that affect the market conditions can be regarded as various random variables that conform to the model. Enterprises can quantitatively analyze the flow among customer categories in the marketing forecast by using the Markov model. In a marketing forecast, businesses can see how many customers they can provide for each type of customer. At the same time, what categories of customers and quantities should be added to provide a more accurate decision-making basis for customer hierarchical management, and at the same time, the guidance for new customer development is more practical, which is beneficial for enterprises to optimize customer structure, improve customer management ability, and ensure that marketing objectives are met.

Based on the data reconstructed by wavelet and the original data, the Markov model for forecasting marketing is established, and the forecasting effect of Markov model is explored. The prediction results are shown in Figures 3-5, respectively. Through the empirical results, it can be found that Markov model has a good prediction effect on the training set of wavelet reconstructed data and original data. On the verification set, the prediction effect based on wavelet reconstruction is slightly better than that based on original data. On the test set, the prediction effect based on the reconstructed data of wavelet is obviously better than that based on the original data. Markov model has a good ability to predict marketing data. On the other hand, it shows that wavelet decomposition and reconstruction of marketing data can effectively improve the generalization ability of Markov model and improve the out-of-sample prediction ability of financial time data.

Traditional marketing forecasting relies heavily on marketing planners' experience as well as qualitative analysis and evaluation by sales staff to predict the future development of a company's overall situation. Although statistical data is occasionally used for overall forecasting, it focuses on the balance and growth of total customers, ignoring the optimization and management of customer structure and the dynamic flow process among different customer categories. If the current sales volume is known, we can guess the sales volume at a certain time in the future without considering the past sales volume, so we can apply Markov forecasting method to make accurate sales forecast. You can also collect the data of other forecast objects, such as the 


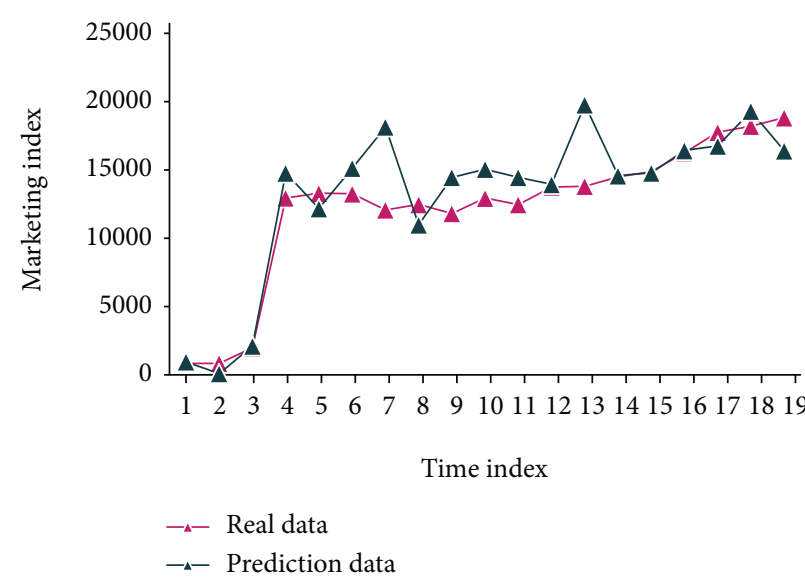

(a)

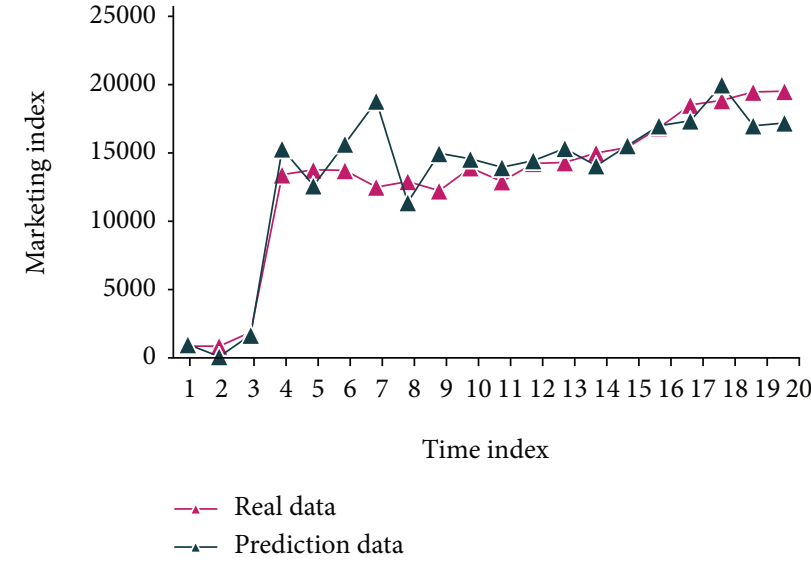

(b)

FIGURE 3: (a) The prediction effect of the training set (wavelet reconstruction data). (b) The prediction effect of the training set (original data).

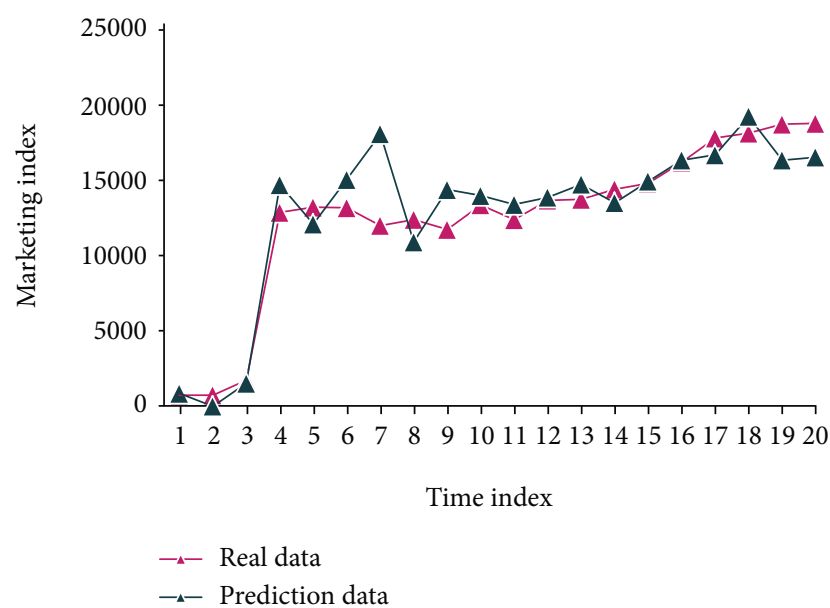

(a)

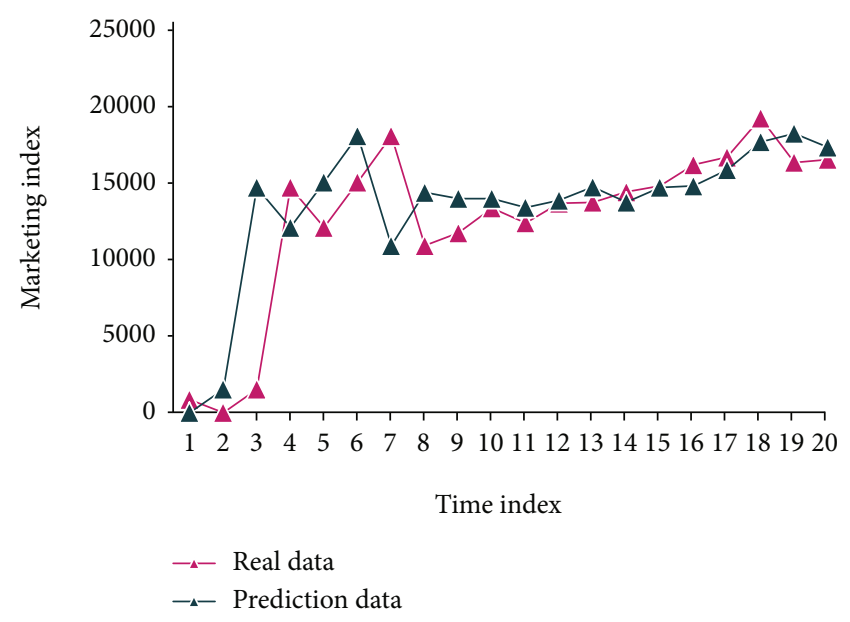

(b)

FIGURE 4: (a) Validation set prediction effect (wavelet reconstruction data). (b) Validation set prediction effect (original data).

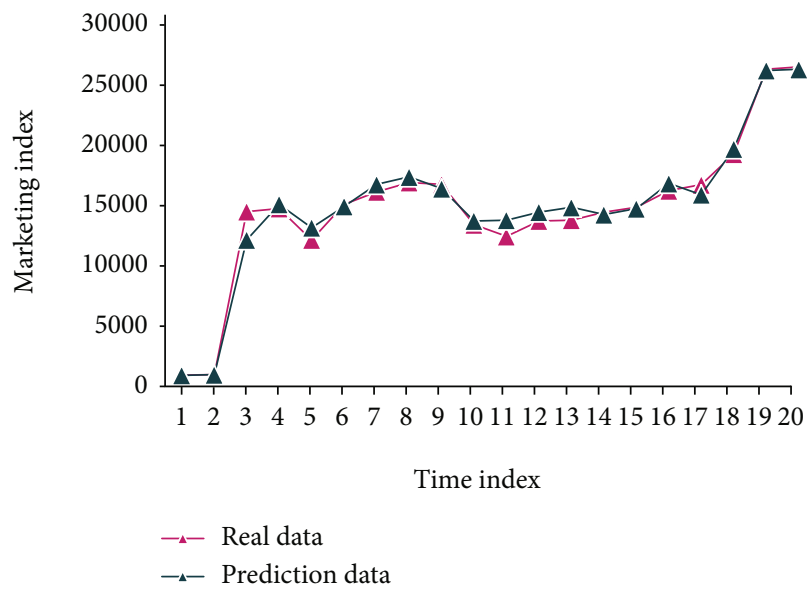

(a)

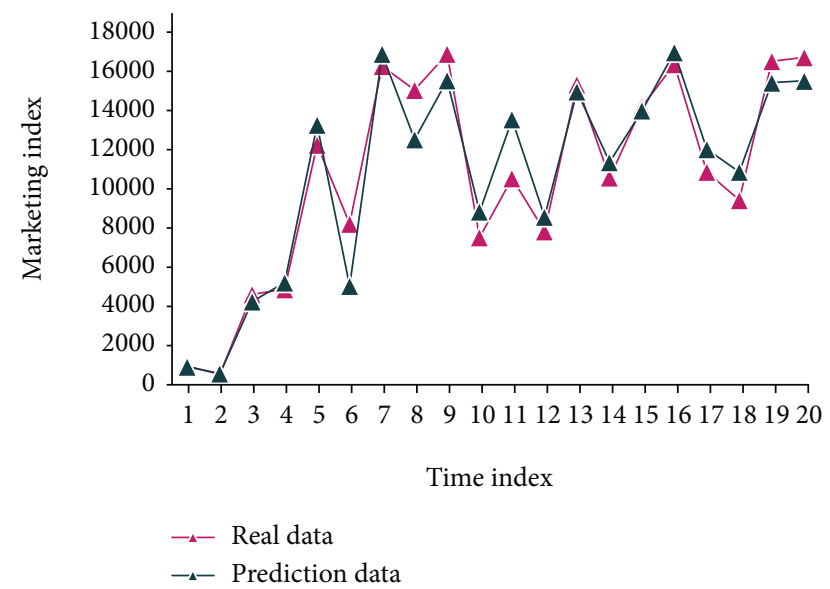

(b)

Figure 5: (a) The prediction effect of the test set (wavelet reconstruction data). (b) Test set prediction effect (original data). 


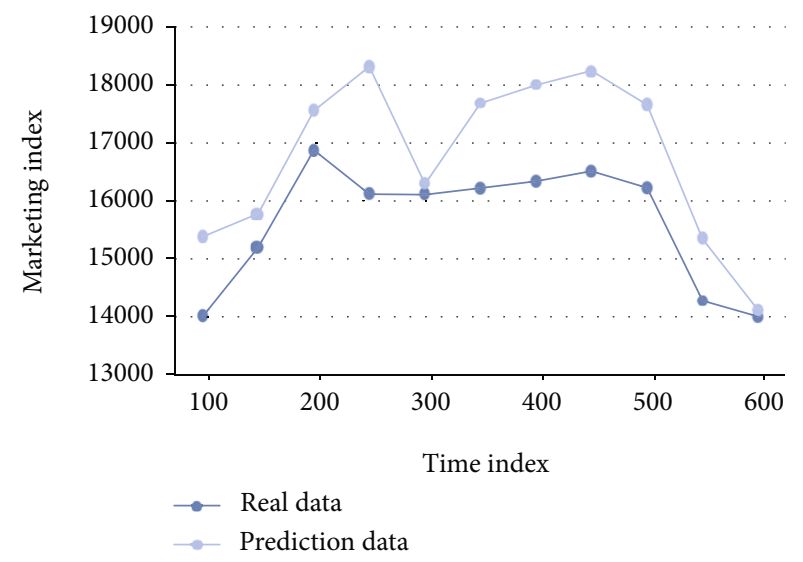

(a)

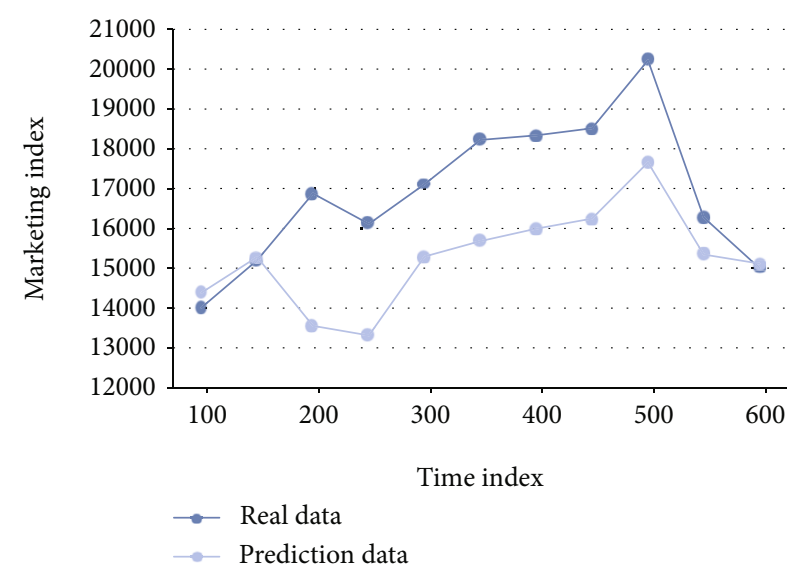

(b)

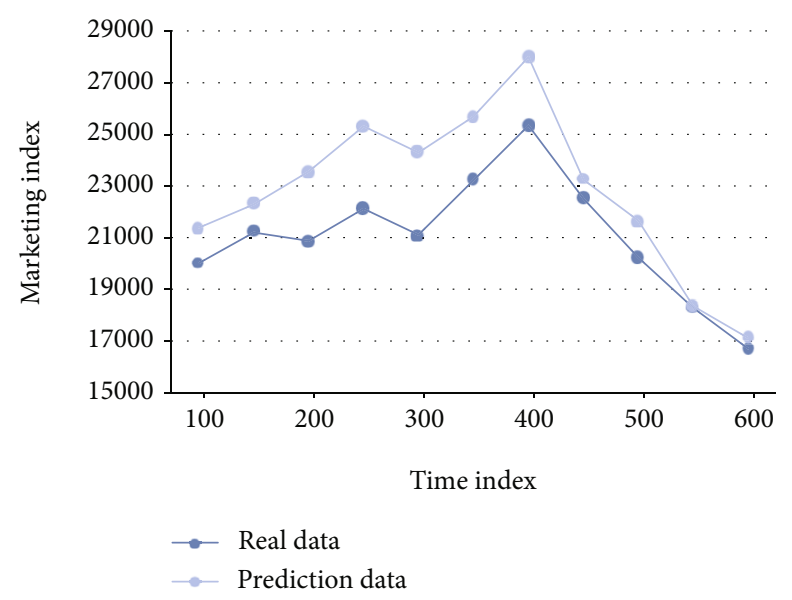

(c)

Figure 6: (a) Dynamic prediction effect (training set). (b) Dynamic prediction effect (validation set). (c) Dynamic prediction effect (test set).

categories of customers' purchases and the number of customers owned by various commodities, for effective analysis.

Marketing forecast is a very important work for enterprises, which determines whether enterprises can survive and grow steadily. Customer changes are highly volatile and random. The forecasting model constructed by Markov chain only needs the current data and does not need to collect a large amount of historical data to forecast the customer supply and changes. The operation method is simple and easy, which can provide quantitative reference for enterprise marketing forecasting and guide enterprises to make more accurate marketing forecasting. Figure 6 shows the dynamic prediction effect of market situation. Generally speaking, Markov model can effectively predict the dynamic trend of market situation in a period of time, regardless of the training set and verification set in the sample or the test set outside the sample. Prove the applicability and effectiveness of Markov model in forecasting marketing situation.

The previous information of the marketing process can be statistically analyzed according to the requirements, so it meets the application conditions of Markov model. For example, when analyzing the accumulated sales of a certain product, the sales at the present moment are definitely known, so the accumulated sales at a certain moment in the future have nothing to do with the accumulated sales at any moment before the present moment. It is very important to accurately predict the products that may be dominant in the future, that is, to predict the purchasing tendency of customers, for making correct business decisions. We can use the Markov process to analyze the number of existing customers who leave our company and go to other companies over time, as well as the number who stay with us, to create a customer transfer matrix, and then predict future changes in customer loyalty, allowing us to develop strategies for customers who have a tendency to transfer ahead of time and keep them as loyal customers. The concept of state transition is introduced in the Markov analysis model so that the probability of transition from one state to another can be calculated, the future can then be judged, and future work can be predicted and guided.

\section{Conclusions}

Many market factors have an impact on the marketing effect. The relationship between market supply and demand, customers' purchase intentions, national financial policies, and other factors is constantly changing, affecting the marketing effect at the same time. Markov model prediction is a 
method based on probability theory and stochastic process theory that analyzes the quantitative relationship in the development and change of real activities using a stochastic mathematical model. The likelihood of each state of an economic phenomenon after multiple transitions is largely determined by how the state transitions, rather than the system's initial state. As a result, a lot of key work is done to measure the transition probability of the system state in order to accurately predict the future state of the phenomenon based on judging the current state of the phenomenon. Because the Markov chain has "no aftereffect," it can be used to forecast market share under the assumption that the market mechanism is dominant. Market share, equilibrium state, and product sales volume can all be predicted using the Markov analysis method. Decision makers can take effective measures to win customers and increase market share and sales volume based on the forecast results. The equilibrium calculation of the user transition probability matrix can be used by decision-makers to obtain the user occupancy rate in an equilibrium state, weigh the benefits and drawbacks, and finally decide whether to shut down and transfer. This paper's proposed Markov model forecasting methods are all suitable for short- and medium-term forecasting. It remains to be seen whether it can be extended to long-term forecasting while maintaining the same forecasting accuracy.

\section{Data Availability}

The data used to support the findings of this study are included within the article.

\section{Conflicts of Interest}

The authors declare that they have no conflicts of interest.

\section{Acknowledgments}

The study was supported by Research on the construction of service system for the electronic commerce in medicine under the background of "Internet + Healthcare" (Grant no. 2021JLDS82).

\section{References}

[1] T. D. Karmarkar, A. Maurer, M. L. Parks et al., "A fresh perspective on a familiar problem," Medical Care, vol. 55, no. 12, pp. 993-1000, 2017.

[2] Z. Hongyu and T. Zhi, "Target track matching method based on hidden Markov model," Ship Electronic Engineering, vol. 306, no. 12, p. 44-47+62, 2019.

[3] T. Chuanzhi and Z. Yuexu, "User cold start recommendation system based on random forest-Markov," Computer Engineering and Design, vol. 407, no. 11, pp. 102-106, 2020.

[4] E. Kappe, A. S. Blank, and W. S. Desarbo, "A random coefficients mixture hidden Markov model for marketing research," International Journal of Research in Marketing, vol. 35, no. 3, pp. 415-431, 2018.

[5] E. Ascarza, O. Netzer, and B. Hardie, "Some customers would rather leave without saying goodbye," Marketing Science, vol. 37, no. 1, pp. 54-77, 2018.
[6] Q. Yanlei, D. Yongping, and Z. Dongyue, "Markov position prediction method based on Gaussian analysis," Computer Technology and Development, vol. 249, no. 1, p. 47-50+56, 2018.

[7] L. Yang, W. Zhenghua, and M. Jie, "A comparative study on the volatility of A-share market stratification index under the Markov system transition," Economic Perspective, vol. 273, no. 6, pp. 46-54, 2018.

[8] J. Di, Y. Xinxin, L. Yuesen, and He Dongxiao, "An efficient Markov random field community discovery method enhanced by structural features," Chinese Journal of Computers, vol. 444, no. 12, pp. 233-247, 2019.

[9] D. Chaoran, L. Sanming, W. Shuai, and P. Zhaoxu, "Fault diagnosis of wind turbine gearbox based on improved continuous hidden Markov model," Journal of Electric Power, vol. 148, no. 1, pp. 73-83, 2019.

[10] C. Ziyang, R. Guoquan, and Z. Yin, "Obstacle recognition in vegetation environment based on Markov random field," Progress in Laser and Optoelectronics, vol. 638, no. 3, pp. 153-162, 2019.

[11] Z. Yang, "Improved adaptive IMM algorithm based on hidden Markov model," Journal of Naval Aeronautical Engineering Institute, vol. 163, no. 6, p. 36-43+77, 2018.

[12] B. Jindong, Z. Jianwei, Z. Qixin, Z. Sandi, and Z. Junlian, "Calculation of UPS reliability index based on Markov process," Electronic Product Reliability and Environmental Testing, vol. 35, no. 4, pp. 25-30, 2017.

[13] T. Yinying, L. Long, and Q. Yang, "Highway freight price prediction based on improved grey Markov model," Journal of Traffic and Transportation Engineering and Information, vol. 59, no. 1, p. 42-47+52, 2018.

[14] G. Weiping, X. Liancheng, R. Min, and L. Fang, "Network forensic evidence fusion method based on parameter optimization hidden Markov model," Application Research of Computers, vol. 33, no. 9, pp. 2778-2781, 2016.

[15] Y. Yuan, Z. Jia, and C. Yi, "Research on monitoring model of price manipulation behavior based on quantitative features," System Engineering Theory and Practice, vol. 36, no. 11, pp. 2721-2736, 2016.

[16] C. Lin, L. Manjun, H. Jian et al., "Power system cascading failure analytical model and probability calculation method based on Markov process," Power System Technology, vol. 41, no. 1, pp. 130-136, 2017.

[17] Liang Xiao, Qian Zhi-Hong, Tian Hong-Liang, and Wang Xue, "Markov decision model based handoff selection algorithm for heterogeneous wireless networks," Acta Physica Sinica, vol. 65, no. 23, pp. 236402-236214, 2016.

[18] L. Ying, K. Lina, W. Yang, and Y. Wu, "Super-element reliability modeling technology under the Markov process of $\mathrm{CNC}$ machine tools," Mechanical Science and Technology, vol. 35, no. 2, pp. 247-252, 2016.

[19] L. Xiang, L. Yong, C. Pengbin, W. Jiewei, and Z. Han, “A new suboccipital undisturbed sleep monitoring scheme based on hidden Markov model [J]," Journal of Biomedical Engineering, vol. 35, no. 2, pp. 280-289, 2018.

[20] H. Weigang and W. Huifang, "Time series anomaly detection method based on autoencoder and hidden Markov model," Journal of Computer Applications, vol. 357, no. 5, pp. 93-98, 2020.

[21] Q. Qian, W. Bingnan, X. Maosheng, F. Xikai, and J. Shuai, "High resolution SAR image building segmentation algorithm 
based on coherence coefficient-Markov random field," Test Science and Instruments: English Edition, vol. 3, pp. 226-235, 2019.

[22] C. Bingcai, W. Xibao, Y. Chao et al., "Saliency detection based on surrounded state and Markov model," Computer Science, vol. 45 , no. 10, p. 272-275+312, 2018.

[23] G. Jian and H. Da, "Analysis of fatigue state of steel box girder orthotropic plate based on Markov chain," China Civil Engineering Journal, vol. 53, no. 3, pp. 60-66, 2020.

[24] X. B. Jin, W. Z. Zheng, J. L. Kong et al., "Deep-learning forecasting method for electric power load via attention-based encoder-decoder with Bayesian optimization," Energies, vol. 14, no. 6, p. 1596, 2021.

[25] H. Qixiang, "Dynamic decision-making problem of investment portfolio in hidden semi-Markov market," Applied Mathematics, vol. 32, no. 1, pp. 51-68, 2019.

[26] J. Valinejad, M. Marzband, M. F. Akorede, T. Barforoshi, and M. Jovanović, "Generation expansion planning in electricity market considering uncertainty in load demand and presence of strategic GENCOs," Electric Power Systems Research, vol. 152, no. 11, pp. 92-104, 2017.

[27] F. Benyu and W. Aiyin, "Application of Markov chain in market forecasting," Cooperative Economy and Technology, vol. 1, no. 1, pp. 56-56, 2017. 